\title{
MageA2 restrains cellular senescence by targeting the function of PMLIV/p53 axis at the PML-NBs
}

\author{
LY Peche ${ }^{1}$, M Scolz ${ }^{1}$, MF Ladelfa ${ }^{2}$, M Monte ${ }^{*, 2}$ and C Schneider ${ }^{*, 1,3}$
}

\begin{abstract}
MAGE-A genes are a subfamily of the melanoma antigen genes (MAGEs), whose expression is restricted to tumor cells of different origin and normal tissues of the human germline. Although the specific function of individual MAGE-A proteins is being currently explored, compelling evidence suggest their involvement in the regulation of different pathways during tumor progression. We have previously reported that MageA2 binds histone deacetylase (HDAC) 3 and represses p53-dependent apoptosis in response to chemotherapeutic drugs. The promyelocytic leukemia (PML) tumor suppressor is a regulator of p53 acetylation and function in cellular senescence. Here, we demonstrate that MageA2 interferes with p53 acetylation at PMLnuclear bodies (NBs) and with PMLIV-dependent activation of p53. Moreover, a fraction of MageA2 colocalizes with PML-NBs through direct association with PML, and decreases PMLIV sumoylation through an HDAC-dependent mechanism. This reduction in PML post-translational modification promotes defects in PML-NBs formation. Remarkably, we show that in human fibroblasts expressing RasV12 oncogene, MageA2 expression decreases cellular senescence and increases proliferation. These results correlate with a reduction in NBs number and an impaired p53 response. All these data suggest that MageA2, in addition to its anti-apoptotic effect, could have a novel role in the early progression to malignancy by interfering with PML/p53 function, thereby blocking the senescence program, a critical barrier against cell transformation.
\end{abstract}

Cell Death and Differentiation (2012) 19, 926-936; doi:10.1038/cdd.2011.173; published online 25 November 2011

Melanoma antigen gene (MAGE)-A proteins belong to the type I MAGE genes subfamily whose expression is restricted to tumor and germinal cells. ${ }^{1}$ Expression of MAGE-A genes is an early event in tumorigenesis and correlates with genomewide hypomethylation, a frequently observed epigenetic event in carcinogenesis. ${ }^{2}$ Owing to their high sequence homology MAGE-A proteins have been considered functionally redundant, and have been largely exploited in the immunotherapy field through cancer vaccine development or as tumor markers. $^{3-5}$ Only during the last few years, their biological function has begun to be investigated. A growing body of evidence indicates that MAGE-A proteins could confer advantages to cancer cells by different mechanisms and with a certain degree of specificity. For instance, MageA1 associates to SKIP and is able to interfere with Notch-IC regulation, ${ }^{6}$ MageA3 is involved in FGFR signaling ${ }^{7,8}$ and MageA11 regulates $A R$ activation. ${ }^{9}$ We have previously demonstrated that MageA2 is a strong inhibitor of the p53 tumor-suppressor transcription factor through histone deacetylase (HDAC)3 recruitment. Thus, in human primary melanoma cells, MageA2 expression confers resistance to chemotherapeutic drugs by interfering with p53 acetylation, which can be reverted by HDAC inhibitor drugs. ${ }^{10}$ Subsequently, other groups also described an opposite correlation between MAGE-A gene expression and p53 activity. ${ }^{7,11,12}$
Interestingly, only MageA4 has been shown to be involved in some potentially anti-tumor functions such as gankyrin oncoprotein inhibition ${ }^{13}$ and apoptosis induction. ${ }^{14,15}$

It has been demonstrated that escape to cellular senescence is one of the first barriers to be bypassed during transformation. ${ }^{16}$ The promyelocytic leukemia (PML) tumorsuppressor protein triggers senescence in normal cells and it has been shown to be involved in oncogenic RasV12-induced senescence. ${ }^{17,18} \mathrm{PML}$ is responsible for the formation of nuclear macromolecular complexes, termed PML-nuclear bodies (PML-NBs). ${ }^{19,20}$ p53 tumor suppressor is recruited to PML-NBs where it became acetylated and activated, and participate in the triggering of cellular senescence. ${ }^{17,18,21,22}$ In addition, PML itself is regulated by acetylation and subsequent sumoylation, ${ }^{23}$ and PMLIV sumoylation has been shown to be required for full p53 activation at the PMLNBs. ${ }^{21,24}$

Here, we have analyzed the ability of MageA2 to interfere with cellular senescence as the final readout of PMLIV activity on p53 acetylation and function. We demonstrate that MageA2 expression correlates with decreased p53 acetylation and activation as induced by PMLIV. Moreover, MageA2 accumulates in PML-NBs through direct interaction with PML proteins and, MageA2 expression results in impaired PMLIV sumoylation and aberrant NB formation. Furthermore, we

${ }^{1}$ Laboratorio Nazionale del Consorzio Interuniversitario per le Biotecnologie, Area Science Park, Padriciano 99, Trieste 34149, Italy; ${ }^{2}$ Departamento Química Biológica, FCEN, Universidad de Buenos Aires, 1428 - Ciudad Universitaria, Buenos Aires, Argentina and ${ }^{3}$ Dipartimento di Scienze e Tecnologie Biomediche, Università di Udine, p.le Kolbe 4, Udine 33100, Italy

${ }^{*}$ Corresponding authors: C Schneider, Laboratorio Nazionale del Consorzio Interuniversitario per le Biotecnologie, Area Science Park, Padriciano 99, Trieste 34149 , Italy. Tel: + 39040375 6804; Fax: + 39040398 990; E-mail schneide @ Incib.it

or M Monte, Departamento Quimica Biologica, FCEN-UBA. Pab-II, Ciudad Universitaria (1428), Buenos Aires, Argentina. Tel: +54 11 45763300, ext 215;

Fax: + 5411 45763342; E-mail mmonte@qb.fcen.uba.ar

Keywords: MAGE; PML; sumoylation; acetylation; p53; senescence

Abbreviations: MAGE, melanoma antigen gene; PML, promyelocytic leukemia; PML-NBs, PML-nuclear bodies; NLS, nuclear localization signal; HDAC, histone deacetylase; TSA, Trichostatin A; SA- $\beta$-Gal, senescence-associated $\beta$-galactosidase activity; SAHF, senescence-associated heterochromatin foci

Received 19.4.11; revised 03.10.11; accepted 24.10.11; Edited by K Vousden; published online 25.11.11 


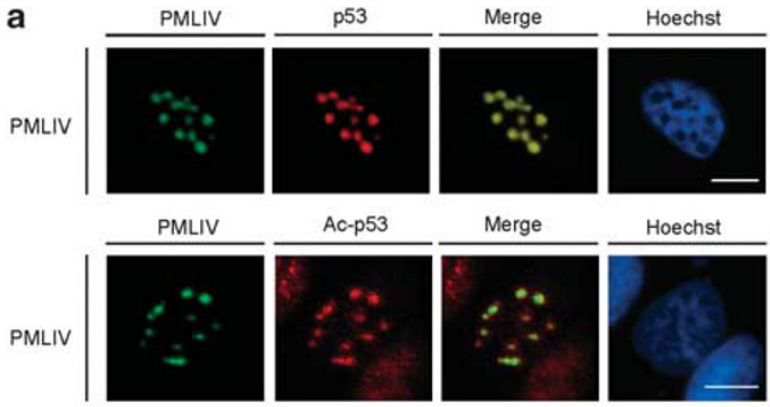

b
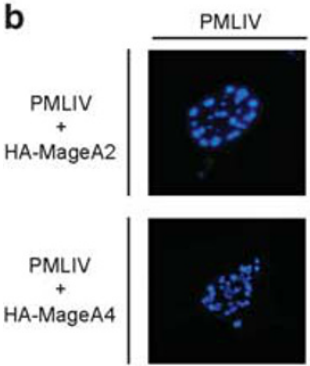

HA
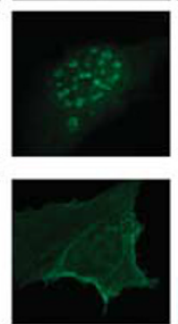

p53

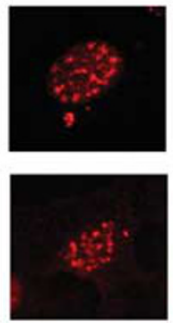

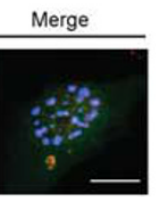

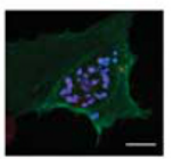

C

PMLIV

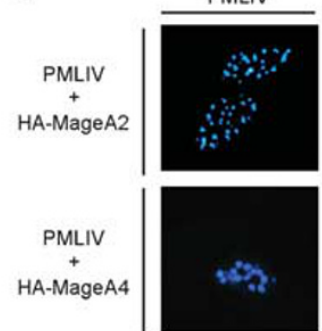

HA

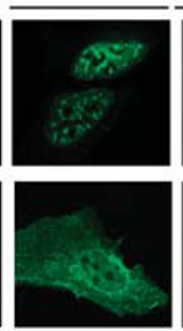

Ac-p53

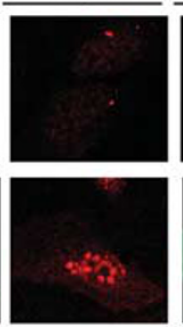

Merge

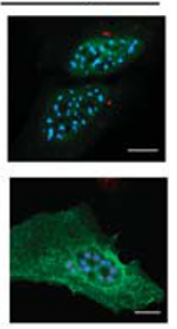

Figure 1 MageA2 represses PMLIV-induced p53 acetylation at PML-NBs. (a) Representative immunofluorescence (IF) images of U2OS cells showing colocalization of transfected PMLIV with endogenous total p53 (upper panel) and acetylated p53 (bottom panel). PMLIV was detected with an anti-PML monoclonal and FITC-conjugated antimouse antibodies, endogenous p53 acetylation status was monitored using an anti-Ac-p53Lys382 and TRITC-conjugated anti-rabbit antibodies. (b) Representative IF images of U2OS cells transfected as indicated, showing colocalization of MageA2, PMLIV and endogenous p53 at PML-NBs. PMLIV was detected with an anti-PML monoclonal and Alexa Fluor 350-conjugated anti-mouse IgG1 antibodies, MageA2 with an anti-HA polyclonal and FITC-conjugated anti-rabbit antibodies, and p53 with DO-I monoclonal and TRITC-conjugated anti-mouse IgG2a antibodies. (c) Panel shows p53 acetylation status at PML-NBs in the same cells of panel B). In this case, PMLIV was detected with an anti-PML monoclonal and Alexa Fluor 350-conjugated anti-mouse IgG1 antibodies, MageA2 with an anti-HA monoclonal and FITC-conjugated anti-mouse IgG2b antibodies, and acetylated p53 with an anti-Ac-p53Lys382 polyclonal and TRITC-conjugated anti-rabbit antibodies. Scale bar: $5 \mu \mathrm{m}$

address the effect of MageA2 in oncogene activated PMLdependent senescence, showing that MageA2 interferes with RasV12-induced cellular senescence and cooperates in cell proliferation, by controlling NBs number and by downregulating the p53-dependent transcriptional activation.

\section{Results}

MageA2 impairs p53 acetylation at PML-NBs. Different kinds of stimuli regulate p53 functions by inducing its posttranslational modifications thus leading to p53 stabilization and activation. ${ }^{25}$ We have previously demonstrated that upon DNA damage MageA2 expression hampers the apoptotic response of cells by affecting p53 acetylation and transactivation function. ${ }^{10}$ PMLIV is a known regulator of p53; indeed it has been shown that PMLIV by recruiting p53 to PML-NBs facilitates its acetylation inducing its transcriptional activity. ${ }^{18,21}$ In this context, we asked whether MageA2 could also affect p53 acetylation as induced by PMLIV. To address this issue, we co-expressed PMLIV and MageA2 in U2OS cells and evaluated the acetylation status of endogenous p53 at PML-NBs. Immunofluorescence analysis showed that PMLIV re-localized p53 to PML-NBs where p53 is present in its acetylated form (Figure 1a). Of note, MageA2 but not MageA4 colocalized with endogenous p53 at NBs (Figure 1b). As MAGE-A proteins share high level of sequence homology, we used MageA4 as specificity control. Indeed, MageA4 behaves differently respect to MageA2, with respect to p53 regulation (Supplementary Figures S1A and $\mathrm{S} 1 \mathrm{C})$. Importantly, expression of MageA2 correlated with a strong reduction of p53 acetylation at NBs, while MageA4 did not affect p53 acetylation status (Figure 1c).

In order to confirm the involvement of MageA2 in interfering with PMLIV-induced p53 acetylation, we knocked-down endogenous MAGE-A and analyzed p53 acetylation upon expression of PMLIV. We observed that cells expressing low levels of MAGE-A showed an increased rate of p53 acetylation by PMLIV, with respect to control cells (Figure 2a). In contrast, when MageA2 was co-expressed along with PMLIV we could corroborate its ability to decrease PMLIV-dependent p53 acetylation (Figure 2b). Finally, as p53 acetylation and recruitment to PML-NBs correlate with increased transcriptional activity of p53, we speculated that MageA2 by dampening p53 acetylation at NBs could affect p53 transactivation function induced by PMLIV. We performed reporter gene assays using the pig3 gene promoter (PIG3-LUC), which was reported to be highly responsive to p53 activation by PMLIV ${ }^{21}$ (Figure 2c, left). Co-expression of MageA2, but not MageA4, resulted in a strong repressive effect on PMLIV-induced p53 activation (Figure 2c, right). It is worth mentioning that the noticed difference between MageA2 and MageA4 on p53 repression seems to be unrelated to their differences in cellular distribution, because a forced nuclear version of MageA4 through nuclear localization signal (NLS) tagging, did not modify its p53 repressing activity or binding capability (Supplementary Figures S1B, S1C and S1D). Altogether, these results suggest that MageA2 localizes to PML-NBs and represses PMLIV-induced p53 acetylation and activation. 

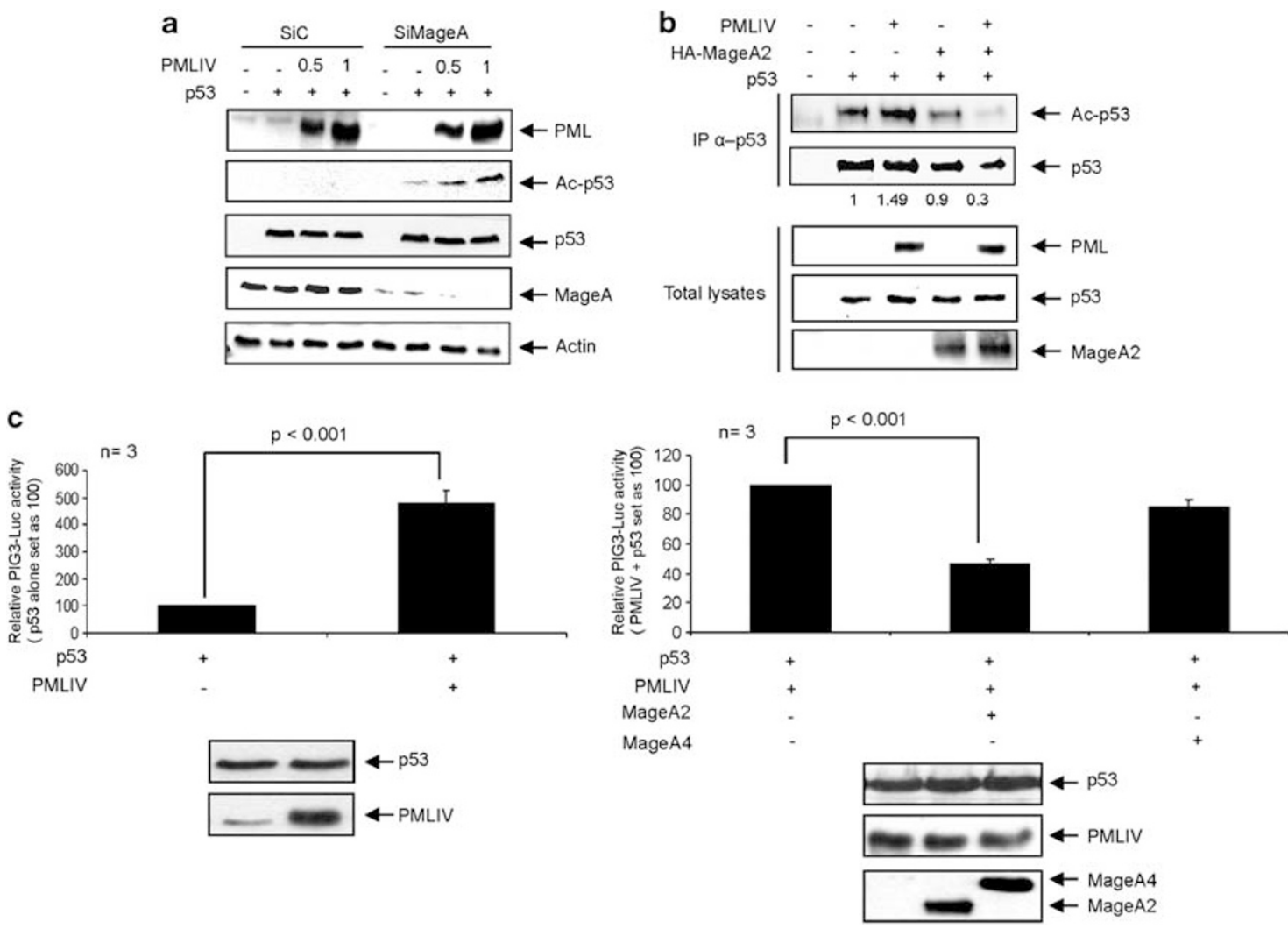

Figure 2 MageA2 represses PMLIV-induced p53 acetylation and function. (a) Analysis of p53 acetylation in H1299 cells transfected as indicated and expressing increasing amounts of PMLIV ( $0.5 \mu \mathrm{g}$ and $1 \mu \mathrm{g})$. Expression of PMLIV, p53 and MAGE-A was analyzed by western blot. Actin levels were used as loading control. (b) H1299 cells were transfected as indicated. p53 was immunoprecipitated with an anti-p53 monoclonal antibody. Total and acetylated levels of immunoprecipitated p53 were analyzed by western blot using anti-p53 and specific anti-Ac-p53Lys382 polyclonal antibodies, respectively. Expression of PMLIV, p53 and MageA2 was evaluated by immunoblotting of total lysates. Numbers below p53 IP blot represent quantification of bands and refer the fold increase in acetylated to total precipitated p53 (acetyl/total p53) ratio, p53 alone was set as 1. (c) Upper: luciferase gene reporter assay in $\mathrm{H} 1299$ cells transfected as indicated. Values corresponding to p53 transfection was reported as 100 (left). Values corresponding to p53 + PMLIV transfection was reported as 100 (right). Lower panels show western blot analysis of lysates used in the luciferase assays. $n$, refers to the number of independent experiments carried out; error bars indicates S.D.; a Student's $t$-test was used for statistical analysis

MageA2 interacts with PMLIV. In order to further characterize MageA2-PML functional interaction, we analyzed the subcellular localization of selected MAGE-A subfamily members. Immunofluorescence assays revealed that MageA2, but not other MAGE-A proteins displays punctuate nuclear staining (Figure 3a). Moreover, extraction of soluble proteins with detergent containing buffer evidenced endogenous MageA2 speckled staining and partial colocalization with endogenous PML bodies in 13923M human melanoma cells that express almost exclusively MageA2 protein and in U2OS cells that express different Mage-A proteins including MageA2 (Figure 3b).

We next evaluated whether MageA2 localization to PMLNBs was dependent on MageA2-p53 interaction. ${ }^{10}$ To this aim, we knocked-down p53 expression in U2OS cells and analyzed MageA2 localization in PMLIV/MageA2 co-transfected cells. Interestingly, MageA2 efficiently colocalized with PMLIV at NBs independently of p53 expression (Figure 3c). Similar results were obtained by co-expressing PMLIV and MageA2 in H1299 p53 null cells (data not shown).

To verify whether PMLIV directly associates with MageA2, we performed an in vitro pull down assay between recombinant GST, GST-MageA2 and GST-MageA4 and in vitro translated PMLIV. Results indicate that MageA2 but not MageA4 interacts directly with PMLIV (Figure 4a).

Interaction between endogenous PML and MAGE-A proteins was evaluated in U2OS cells (Figure 4b, left). Owing to high sequence homology among MAGE-A proteins, antibodies generally recognize more than one MAGE-A subfamily member. Hence, Figure $4 \mathrm{~b}$ shows that at least one or more endogenous MAGE-A proteins form complexes with PML in U2OS cells. Similar results were obtained by immunoprecipitation assay performed in 13923M melanoma cells (Figure $4 b$, right), thus strengthening the fact that endogenous MageA2 and PML interacts in cells. Then, we carried out immunoprecipitation assay after overexpressing both PMLIV and HA-MageA2 or HA-MageA4 (Figure 4c), confirming a degree of specificity in MageA2 and PMLIV interaction.

As MageA2 colocalizes with endogenous PML-NBs, and different $\mathrm{PML}$ isoforms are expressed in cells as a result of alternative splicing of the $\mathrm{pml}$ gene, ${ }^{26}$ we tested whether MageA2 interacts differentially with PML isoforms. We performed co-immunoprecipitation between different Flagtagged PML isoforms (I to $\mathrm{VI}$ ) and HA-MageA2. As shown in Figure $4 \mathrm{~d}$, MageA2 is able to bind PMLI, II, IV, V and to a 
a
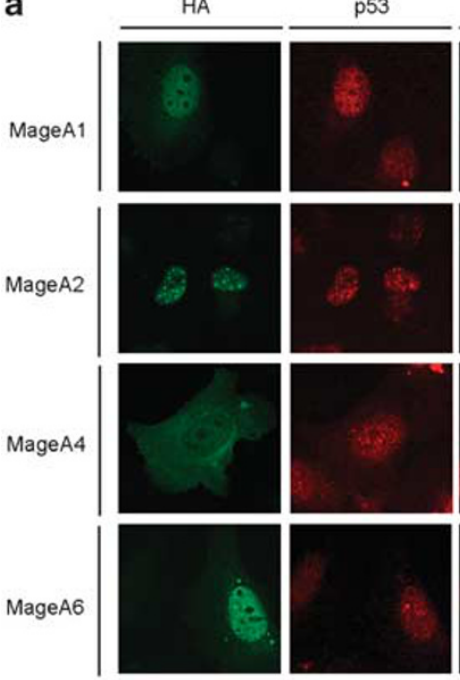

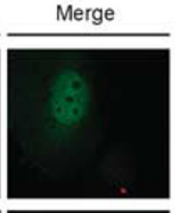

Hoechst

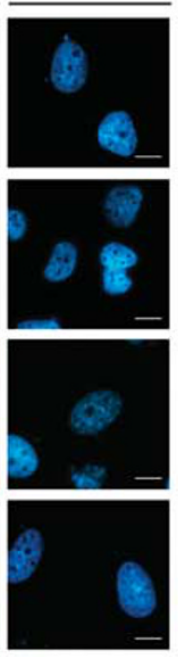

b
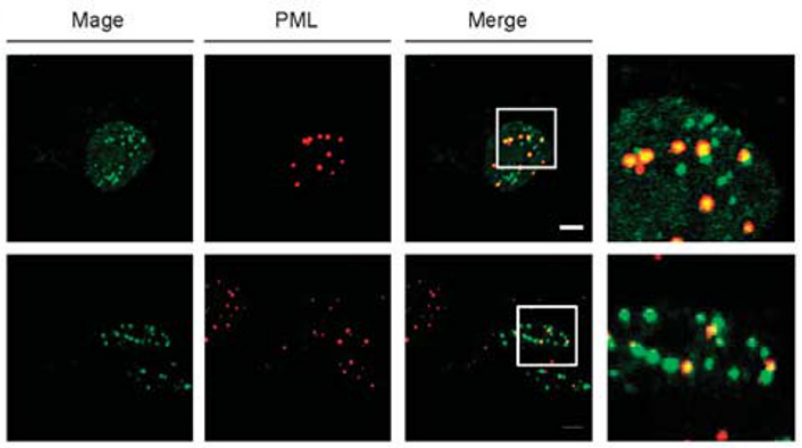

C
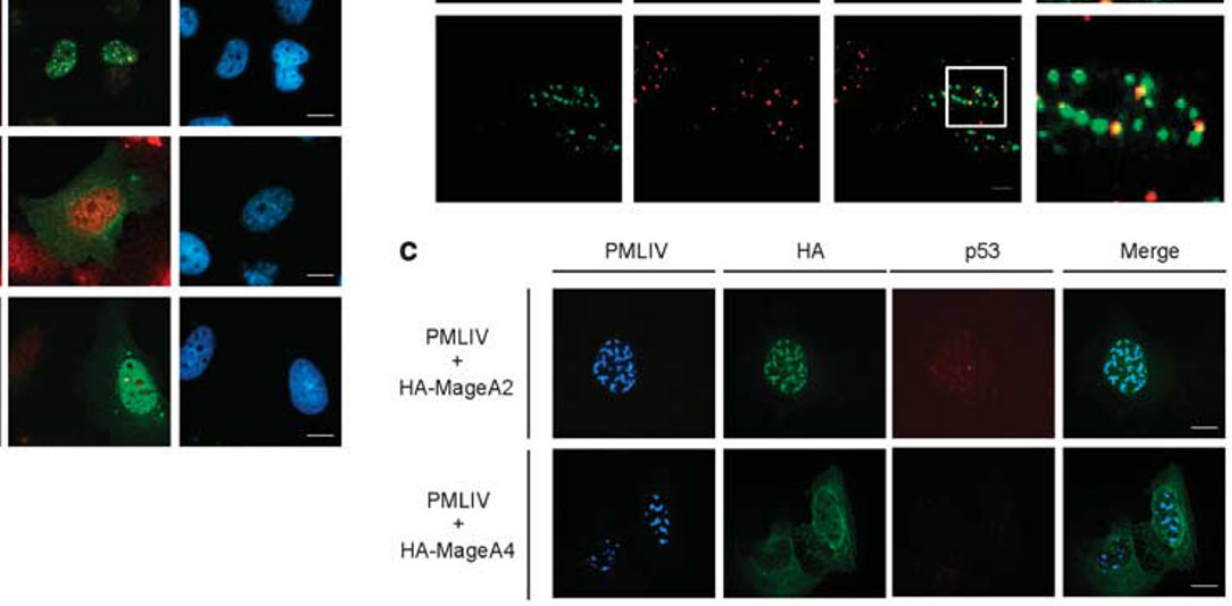

Figure 3 MageA2 specifically localizes at PML-NBs. (a) Representative IF images of U2OS cells transfected with HA-MageA1, -A2, -A4 and -A6, using an anti-HA polyclonal antibody. Endogenous p53 was detected using monoclonal anti-p53 antibody (DO-I). Primary antibodies were recognized with FITC-conjugated anti-rabbit antibody and TRITC-conjugated anti-mouse antibody, respectively. (b) Representative confocal IF images analyzing endogenous MageA2 and PML proteins colocalization in the melanoma cell line 13923M (upper) and HA-MageA2 and endogenous PML in U2OS cells (lower). Cells were treated with CSK buffer before fixation to eliminate insoluble proteins. PML was detected with an anti-PML monoclonal antibody and MageA2 with an anti-MageA polyclonal antibody. Right panels represent higher magnifications of the selected areas in the merge images. (c) Representative IF from U2OS cells transfected with specific siRNA to knock-down p53 and transfected with PMLIV in combination with HA-MageA2 or HA-MageA4. MAGE-A proteins were visualized with an anti-HA monoclonal and FITC-conjugated anti-mouse IgG2b antibodies, PMLIV with an anti-PML polyclonal and Alexa Fluor 350-conjugated anti-rabbit antibodies and p53 with a monoclonal anti-p53 and TRITC-conjugated anti-mouse IgG1 antibodies. Scale bar: $5 \mu \mathrm{m}$

a
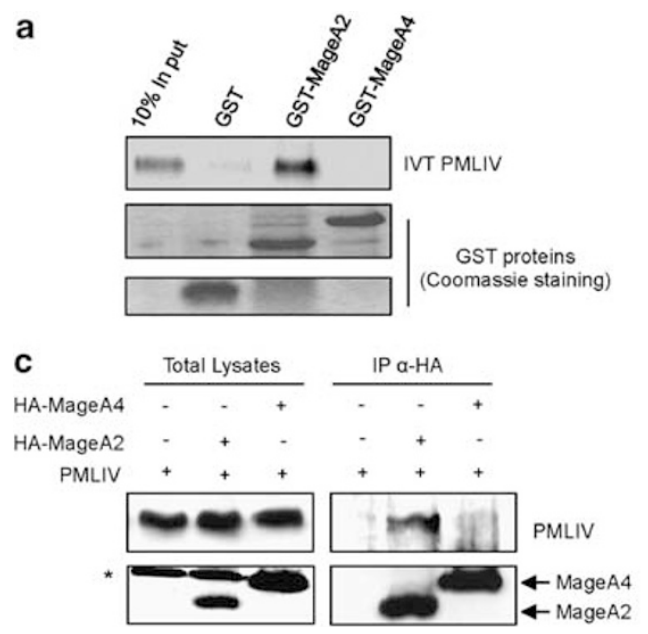

b

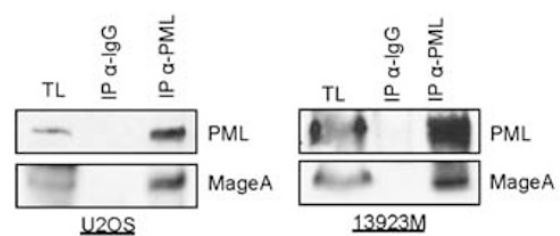

d

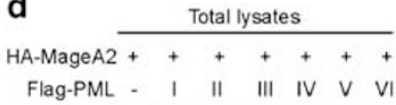

$\frac{\text { IP } \alpha \text {-Flag }}{+++++++}$

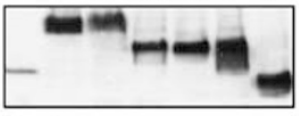

$-3-2-2$
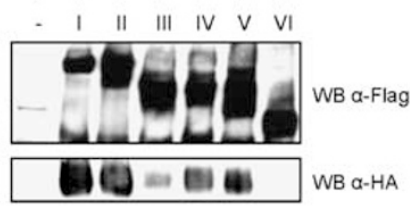

Figure 4 MageA2 binds to PMLIV. (a) In vitro binding assay using recombinant/purified GST, GST-MageA2 or GST-MageA4 fusion proteins incubated with in vitro translated ${ }^{35}$ S-labeled PMLIV (IVT PMLIV). (b) Immunoprecipitation (IP) of endogenous PML/MAGE-A complex in U2OS cells (left) or in the melanoma cell line 13923M (right) using anti-PML monoclonal antibody, control IP was performed with an anti-Flag monoclonal antibody. (c) IP in 293T cells transfected with PMLIV and HA-MageA2 or HAMageA4 using anti-HA monoclonal antibody. (d) IP in 293T cells transfected with HA-MageA2 and different Flag-tagged-PML isoforms using an anti-Flag monoclonal antibody. TL, total lysate. *Unspecific band

lesser extent PMLIII, but no interaction was observed with PMLVI. All these interactions were confirmed by in vitro binding assay (Supplementary Figure S2). In summary, all these data suggest that MageA2 interacts with PML at the PML-NBs in a p53-independent manner. 


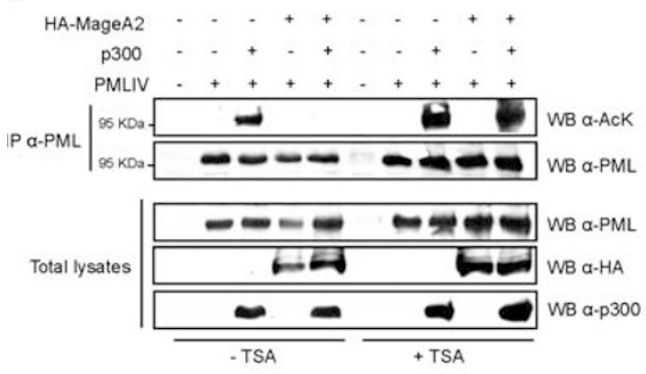

b

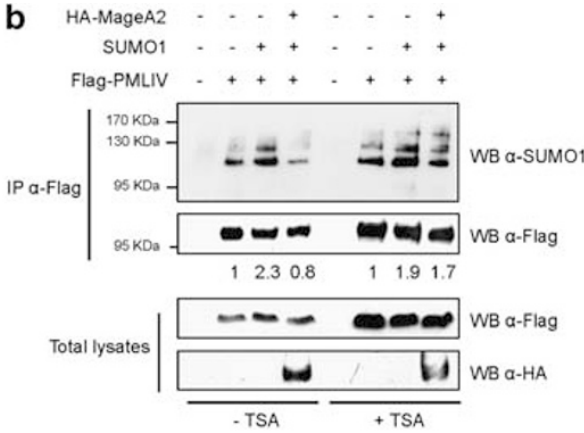

C

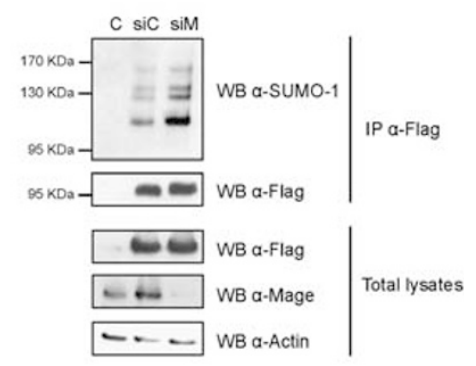

Figure 5 MageA2 impairs PMLIV post-translational modifications. (a) Analysis of PMLIV acetylation in 293T cells transfected as indicated. PMLIV was immunoprecipitated with an anti-PML monoclonal antibody. Acetylated and total PMLIV was detected in western blot with an anti-acetylated lysine and anti-PML polyclonal antibodies, respectively. At $6 \mathrm{~h}$ after transfections, cells were treated or not with $1 \mu \mathrm{M}$ TSA for $24 \mathrm{~h}$. (b) Analysis of PMLIV sumoylation in 293T cells transfected as indicated. PMLIV was immunoprecipitated with an anti-Flag antibody. The membrane was first incubated with an anti-SUMO1 monoclonal antibody to detect sumoylated PMLIV and subsequently with an anti-PML polyclonal antibody to detect total PMLIV. HA-MageA2 was detected with an anti-HA monoclonal antibody. At $6 \mathrm{~h}$ after transfections, cells were treated or not with $1 \mu \mathrm{M} \mathrm{TSA}$ for $24 \mathrm{~h}$. Numbers below Flag blot in the IP represent quantification of bands and refer the fold increase in sumoylated to total precipitated PMLIV (sumo/total PMLIV) ratio, PMLIV alone was set as 1. (c) Analysis of PMLIV sumoylation in U2OS cells stably expressing Flag-PMLIV and transfected with control (siC) or MageA2-specific (siM) siRNA. PMLIV was immunoprecipitated with an anti-Flag monoclonal antibody. Sumoylated and total PMLIV was detected in western blot with anti-SUMO-1 and antiPML polyclonal antibodies, respectively. Line $\mathrm{C}$ corresponds to U2OS control cell line

Although MageA2 is able to interact with different PML isoforms, subsequent experiments investigating the functional relevance of MageA2-PML interaction have been focused on PMLIV, which mediates acetylation and activation of p53.

MageA2 reduces PMLIV acetylation and sumoylation. PML functionality is highly regulated by post-translational modifications such as phosphorylation, acetylation and sumoylation. ${ }^{27}$ Along with others, we have demonstrated that MAGE-A proteins can associate to HDAC. ${ }^{6,10}$ As acetylation has been recently reported to be a prerequisite for subsequent PML sumoylation, ${ }^{23}$ we decided to investigate whether MageA2-PMLIV interaction could affect PMLIV acetylation. To verify this point we co-transfected cells with PMLIV and p300 to stimulate PMLIV acetylation. Then, PMLIV acetylation was detected using anti-acetyl lysine antibody on immunoprecipitated PMLIV. As shown in Figure $5 \mathrm{a}$, expression of MageA2 together with PMLIV/p300 strongly decreased PMLIV acetylation. Importantly, treatment with the HDAC inhibitor Trichostatin A (TSA) reverted the effect of MageA2 expression on PMLIV acetylation, indicating that endogenous HDACs are involved in such effect (Figure 5a). In support of this data, we observed that MageA2 expression induces the recruitment of HDAC3 to PML-NBs (Supplementary Figure S3A).

Moreover, PMLIV reduced acetylation resulted in PMLIV sumoylation defect: PMLIV was efficiently sumoylated in the presence of SUMO1, whereas MageA2 expression significantly reduced such modification and, accordingly to our previous result, the effect was dependent on endogenous HDAC activity (Figure 5b). Importantly, siRNA-mediated downregulation of MageA2 in U2OS cells stably expressing PMLIV resulted in increased levels of sumoylated PMLIV, demonstrating the involvement of endogenous MageA2 in the regulation of PMLIV sumoylation (Figure 5c). Interestingly, sumoylation of PMLVI isoform was not affected by MageA2 expression (Supplementary Figure S3B), demonstrating the relevance of MageA2-PML interaction. Altogether these results suggest that MageA2 could affect PMLIV sumoylation through an HDAC-dependent mechanism.

MageA2 impairs PML-NBs formation. It has been demonstrated that PML sumoylation and protein-protein interactions of PML through SUMO are involved in the proper formation of PML-NBs. ${ }^{20,28}$ As our results indicate that MageA2 negatively regulates PMLIV post-translational modifications, in particular sumoylation, we decided to investigate whether MageA2 could affect PML-NB formation. We used $\mathrm{PML}^{-1-} \mathrm{MEF}$ to analyze PML-dependent neo-formation of NBs. Expression of PMLI, PMLIV or PMLVI in $\mathrm{PML}^{-1-} \mathrm{MEF}$ leads to the formation of NBs that differ in their number and size (Figure 6a, left). Intriguingly, expression of MageA2 affected the typical morphology and the number of PML-NBs formed by PMLI and PMLIV but not by PMLVI, in agreement with the previously demonstrated capability of MageA2 to interact with specific PML isoforms (Figure 6a, middle). Quantification of the number of cells showing normal number (15-30 NBs per nucleus) and morphology (tiny punctuate) of NBs structures in cells co-expressing MageA2 and PMLIV with respect to PMLIV alone, indicates the degree of impairment that MageA2 expression caused on PMLIV-NBs formation (Figure 6a, right). As a measure of correct formation of PML-NBs, we also evaluated the ability of PML to colocalize with SUMO1 at PML-NBs in cells expressing (Figure 6b, middle) or not MageA2 (Figure 6b, left). Importantly, the ability of PMLI and PMLIV to correctly colocalize with SUMO1 at NBs was significantly impaired by MageA2 expression (Figure 6b, middle and right). Expression of SUMO1 alone displayed a diffused nuclear staining pattern because SUMO1 accumulates mainly in PML-NBs, and in $\mathrm{PML}^{-1-}$ cells, PML-NBs do not form (data not shown). In some cases, MageA2 expression blocked colocalization between PML and SUMO1 (Figure 6b, middle), or alternatively it was observed complete colocalization but the overall PML-NB structure was affected by MageA2 expression (Supplementary Figure S4). MageA2 did not affect PMLVI-NBs formation or the 


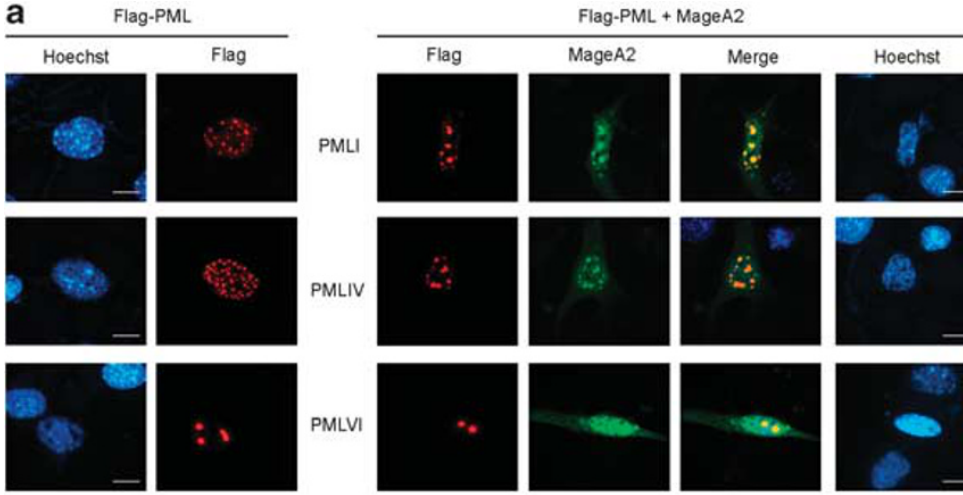

b Flag-PML + GFP-SUMO1
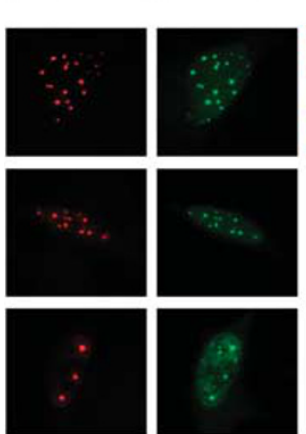
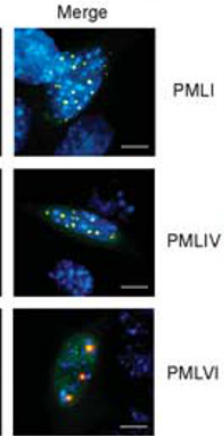

Flag-PML + GFP-SUMO1 + MageA2
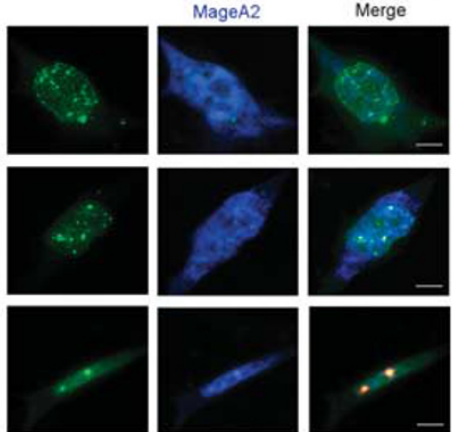

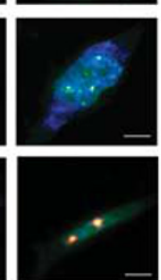

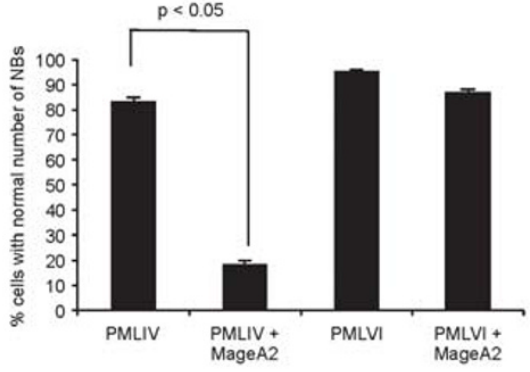

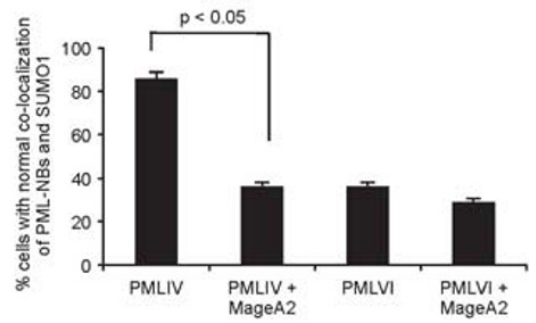

Figure 6 MageA2 impairs nuclear bodies formation. (a) Representative IF images from $\mathrm{PML}^{-1-}$ MEFs transfected with Flag-PML isoforms alone or in combination with HA-MageA2. PML isoforms were detected with an anti-Flag monoclonal and TRITC-conjugated anti-mouse antibodies, and MageA2 with an anti-HA polyclonal and FITCconjugated anti-rabbit antibodies. Graphic on the right represents the percentage of cells showing normal number (15-30 NBs per nucleus) and shape (tiny bodies) of NBs for cells expressing PMLIV or PMLVI alone or in combination with MageA2. At least 200 cells were counted in three independent experiments. (b) Representative IF images from $\mathrm{PML}^{-1-}$ MEFs transfected with Flag-PMLs and GFP-SUMO1 alone or in combination with HA-MageA2. PML isoforms were detected with an anti-Flag monoclonal and TRITCconjugated anti-mouse antibodies, and MageA2 with an anti-HA polyclonal and Alexa Fluor 350-conjugated anti-rabbit antibody. Graphic on the right represents the percentage of cells showing normal colocalization of PML and SUM01 in cells expressing PMLIV or PMLVI alone or in combination with MageA2. At least 200 cells were counted in three independent experiments. Error bars indicate S.D.; a Student's $t$-test was used for statistical analysis. Scale bar: $5 \mu \mathrm{m}$

colocalization between PMLVI and SUMO1 (Figure 6b). Importantly, colocalization of MageA2 and PMLIV at NBs in PML knockout cells was not as efficient as shown in PMLexpressing cells, suggesting that interaction with multiple $\mathrm{PML}$ isoforms could increase MageA2 retention to NBs. Altogether these data indicate that, at least through this overexpression approach, MageA2 could disturb the proper formation of PML-NBs and their overall structure. These effects could be probably attributable to impaired PML posttranslational modifications and the consequent interference with partners recruitment to PMLIV-NBs.

MageA2 impairs cellular senescence by affecting PMLIVinduced p53 activity and PML-NBs formation. A connection between PMLIV overexpression, p53 recruitment to NBs, increased p53 acetylation and induction of premature cellular senescence has been already suggested. ${ }^{17,18,22}$ Given that MageA2 colocalizes with p53 and PMLIV in the NBs inducing p53 deacetylation and repressing its transactivation function, we investigated the impact of MageA2 expression on PMLIV-induced senescence. To this end, normal human fibroblasts were co-transduced using retroviral vectors expressing PMLIV with an empty vector or in combination with MageA2 or alternatively MageA4. After 10 days under selective culture conditions, PMLIV transduced cells showed all the features of the senescence process, because they ceased to proliferate at sub-confluent densities and became flat and enlarged. Importantly, cells co-expressing PMLIV and MageA2 did not show major morphological changes and behaved similarly to control cells (Figure 7a). Colocalization between MageA2 and PMLIV was observed in these cells (data not shown). Analysis of senescenceassociated $\beta$-galactosidase activity (SA- $\beta$-Gal), a well-known marker of senescence, demonstrated that MageA2 expression significantly reduced SA- $\beta$-Gal levels in PMLIV-expressing cells (Figure $7 \mathrm{~b}$ ). Moreover, cells expressing MageA2 and PMLIV showed higher levels of BrdU incorporation with respect to those expressing only PMLIV (Figure $7 \mathrm{~b}$ ). Cells overexpressing MageA2 alone behaved indistinguishably from control or MageA4 transduced cells. Importantly, MageA2 expression correlated with reduced p21 protein levels, probably due to an effect of MageA2 on efficient p53 activation (Figure 7c).

In normal cells, cellular senescence acts as a barrier to prevent transformation triggered by oncogene deregulation. ${ }^{16}$ For instance, cells respond to RasV12 oncogene overexpression by activating a senescence program through a functional PML/p53 axis. ${ }^{17,18}$ In fact, PML has been demonstrated to be involved in RasV12-induced senescence by stimulating p53 acetylation and function. Moreover, the number of PML-NBs 
a

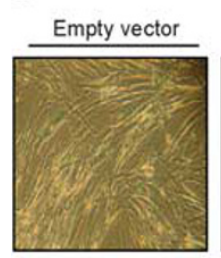

PMLIV

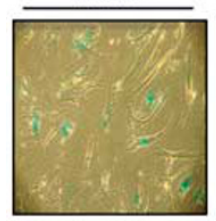

b

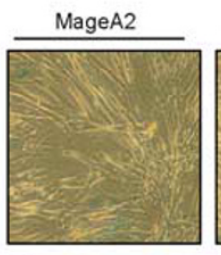

PMLIV + MageA2

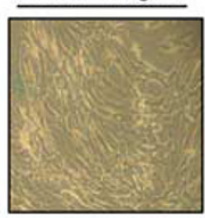

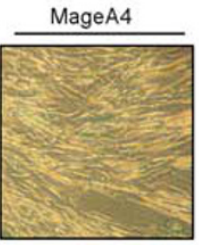

PMLIV + MageA4

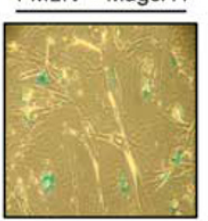

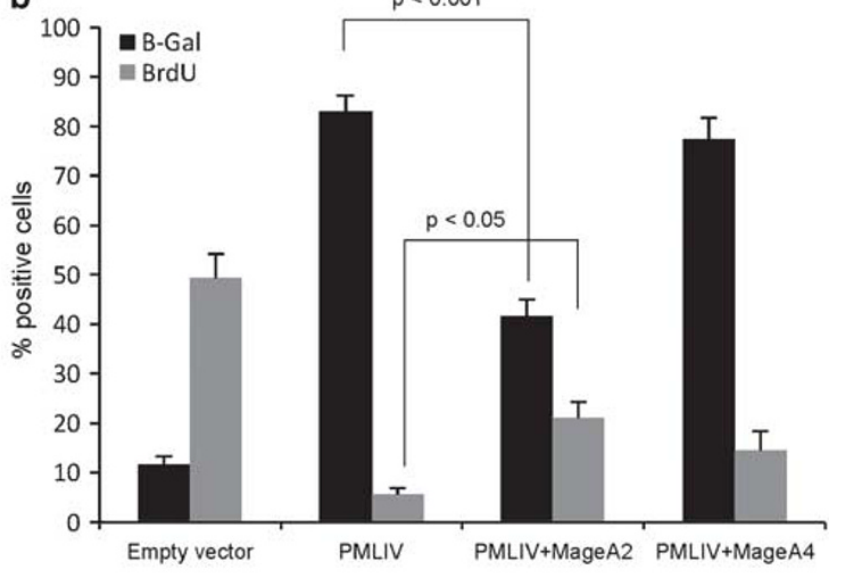

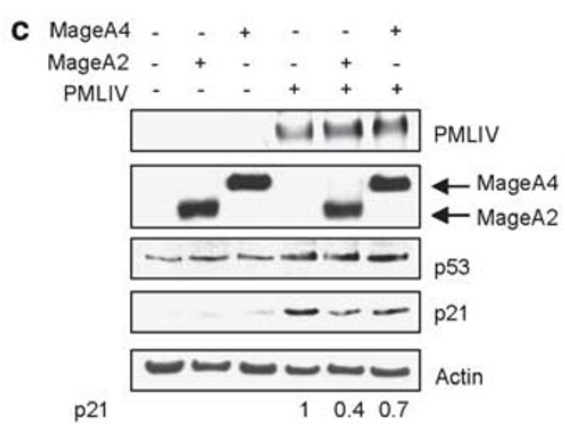

Figure 7 MageA2 antagonizes PMLIV-induced senescence. (a) Wi38 cells were infected with the indicated retroviruses, after 10 days under selection conditions, cells were fixed and stained for SA- $\beta$-Gal activity and photographed under a phase contrast microscopy to analyze cell morphology. (b) Analysis of the percentage of cells that stained positive for SA- $\beta$-Gal and were positive for BrdU incorporation after counting 200 cells in at least three independent experiments is shown. Error bars indicate S.D.; a Student's $t$-test was used for statistical analysis. (c) Western blot analysis of lysates obtained from Wi38 cells used in (a) and (b). Actin was used as loading control. Numbers below Actin blot represent quantification of p21 bands respect to Actin, the p21 value from PMLIV alone infected cells was set as 1

significantly increases during senescence, implying an enhancement in the PML-NB formation process. ${ }^{17,18}$ In this context, we examined whether MageA2 could regulate RasV12-induced senescence. To address this issue, we used primary human BJ fibroblasts expressing hTERT and 4-OHtamoxifen (4-OHT)-inducible oncogenic H-RasV12 (BJ/ET/ RasV12ER cells) ${ }^{29}$ co-transduced or not with MageA2. After induction of $\mathrm{H}$-RasV12 in BJ cells, several markers of premature senescence were observed, such as the increase in SA- $\beta$-Gal-positive cells and the formation of senescenceassociated heterochromatin foci (SAHF) (Figure 8a). Importantly, we observed that MageA2 expression impaired RasV12-induced SA- $\beta$-Gal activity and SAHF formation and resulted in higher levels of BrdU incorporation in those cells (Figure 8a). Expression of MageA2 was unable to regulate senescence parameters when PML was knocked-down by shRNA (Supplementary Figure S5). Moreover, MageA2 expression affected p53 activity in this cellular system, as assessed by quantification of p53 target genes (Figure 8b), thus confirming that MageA2 downregulates the p53-dependent response. Similar results were shown at the protein level; in fact, p21 seemed to be highly sensitive to MageA2 expression when compared with other p53 target proteins (data not shown).

Finally, RasV12-induced formation of PML-NBs during senescence was analyzed. As previously reported, ${ }^{17,18}$ after RasV12 induction the number and size of PML-NBs was increased, nevertheless MageA2 expression significantly blocked this increase (Figure $8 \mathrm{c}$ and Supplementary Figures S6A and S6B). Colocalization between MageA2 and PML was also observed in these cells (Supplementary Figures S6A and S6B). Collectively, all these data support the notion that MageA2 impairs the senescence process by targeting the PML/p53 axis, thus blocking a central barrier against cell transformation and tumor progression.

\section{Discussion}

MAGE proteins have been discovered as tumor antigens and for many years studies have been mainly focused on their use as immunotherapeutic targets in cancer. Moreover, because of high sequence homology, MAGE genes were considered functionally redundant. Nevertheless, in the last years compelling evidence has accumulated suggesting that individual MAGE proteins could behave in specific pathways critical for tumor progression. ${ }^{30-34}$ We previously demonstrated that MageA2 is a relevant player in favoring chemoresistance to p53 bearing human melanoma cells. Here, we analyze the role of MageA2 as regulator of another important tumor-suppressive mechanism, namely cellular senescence. The relevance of p53 acetylation as a trigger of its function has demonstrated to be highly regulated by the PML tumor suppressor. ${ }^{17,18} \mathrm{~A}$ model to explain the physiological role of PML-NBs is based on the assumption that they 
a

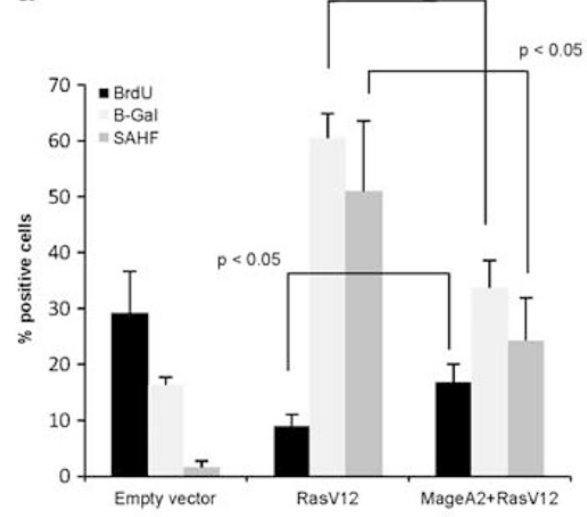

C

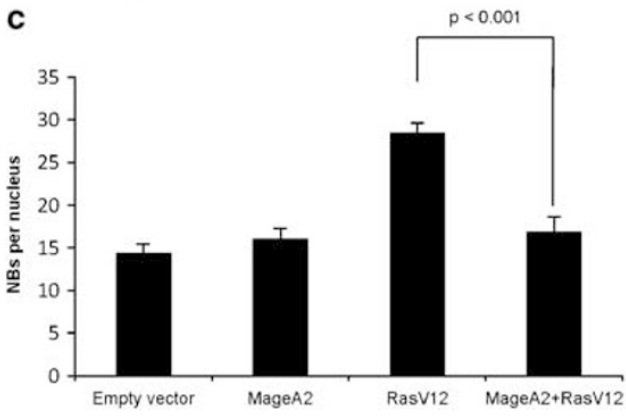

b
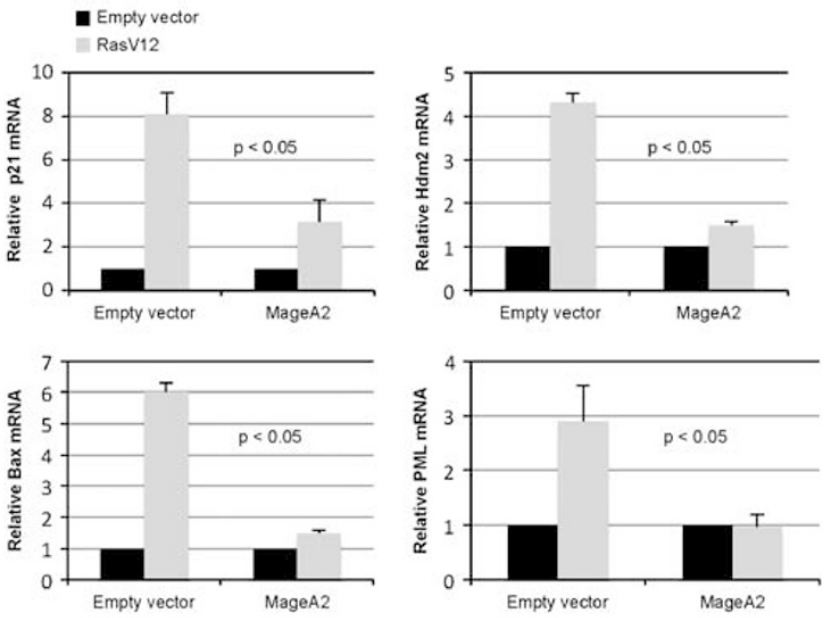

Figure 8 MageA2 antagonizes RasV12-induced senescence. (a) Primary human BJ fibroblasts expressing hTERT and 4-OH-tamoxifen (4-OHT)-inducible oncogenic HRasV12 (BJ/ET/RasV12ER cells) co-transduced in combination with retroviruses expressing MageA2 were induced for 10 days. Subsequently, BrdU incorporation, B-Gal expression and SAHF number were analyzed. Graphic represents average of cells positive for BrdU, B-Gal or SAHF, after counting 200 cells in at least three independent experiments. (b) Quantitative RT-PCR analysis for the indicated mRNAs in cells from (a). Graphs represent mean from at least three independent experiments. (c) Analysis of average number of NB per nucleus in cells from (a) after counting 200 cells in at least three independent experiments. Error bars indicate S.D.; a Student's $t$-test was used for statistical analysis

function as sites for the recruitment of different proteins and their post-translational modification. ${ }^{19}$ Here, we show that a fraction of MageA2 accumulates in PML-NBs and this consequently results in an impairment of p53 acetylation at the NBs and the ability of PMLIV to efficiently induce p53 function. As the colocalization of MageA2 with PML at NBs is not dependent on p53 expression, MageA2 could be able to regulate other $P M L$ functions not involving p53 activity. Besides, the ability of MageA2 to interact with different PML isoforms could improve its localization to NBs and suggests a potential role of MageA2 in the regulation of PML isoformspecific functions.

An important consideration raised by our results is that MageA2 behaves in a specific manner considering its distinguishable cellular localization with respect to other MAGE-A proteins. Importantly, MageA2 specificity was emphasized by the fact that despite their high sequence similarities MageA4 does not behave as MageA2 both in terms of p53 and/or PMLIV regulation, even when MageA4 was targeted with a NLS.

PML functionality itself is highly affected by post-translational modifications, such as phosphorylation, sumoylation and as recently demonstrated acetylation. ${ }^{23,26,27}$ In fact, it has been suggested that p300-dependent acetylation of PML allows its subsequent sumoylation and activation. ${ }^{23}$ Our data suggest that MageA2 could affect directly PMLIV function by interfering with $P M L$ post-translational modifications. We have recently reported that MageA2 forms active complexes with
HDACs to favor deacetylation of $p 53 ;^{10}$ here we show that MageA2 could also be found in PML-NBs impairing PML sumoylation through an HDAC-dependent mechanism. Although it has been reported that PMLIV binds slightly to HDAC3, ${ }^{35}$ MageA2 could enhance HDAC recruitment to PML-NBs, suggesting that MageA2/HDAC3 complexes could be assembled and be functional on different nuclear domains and cellular contexts. This mechanism could explain not only the observed reduction in PML acetylation, but also that of p53 at NBs. Furthermore, although the precise mechanism and final outcome of PML acetylation has not yet been fully addressed, it is possible to hypothesize that different PMLIV functions depending on its acetylation or sumoylation could be impaired in tumor cells expressing MageA2.

PML post-translational modifications, in particular sumoylation, are important in many ways. PML sumoylation is required for proper nucleation of NBs and for recruitment of its partners into NBs. ${ }^{20,28}$ Many oncogenic viral proteins target PML functions by affecting the integrity of the NB through the control of PML sumoylation. ${ }^{26}$ PMLIV stability is not affected by MageA2 expression, considering the reported involvement of PML sumoylation in the regulation of its stability ${ }^{36,37}$ and recent observations that Mage/RING protein complexes could induce ubiquitin-dependent degradation of targeted proteins. ${ }^{38}$ Instead, MageA2 seems to affect PML-NBs proper formation and partner's recruitment as we demonstrated in $\mathrm{PML}^{-1-}$ MEFs. Moreover, induction of $\mathrm{PML}$ by oncogenic RasV12 is not affected by MageA2, nevertheless the overall 
structure and the number of NBs upon RasV12 expression is clearly affected by MageA2, suggesting a defect in the assembly of NBs and not on its stability.

Sumoylation-deficient PMLIV is not able to regulate p53 function and SUMO-modified PML results in transactivation and strong recruitment of p53 to NBs. ${ }^{21}$ p53 accumulation at PML-NBs is accompanied by induction of p53 acetylation: a hallmark for PMLIV-induced senescence is the formation of a functional complex between p53, CBP and PMLIV, which results in acetylation and transcriptional activation of p53, followed by growth arrest and senescence. ${ }^{17,18}$ It has been previously reported that factors affecting p53 acetylation at NBs, such as $\operatorname{Sirt}^{39}$ or viral oncoproteins like $E 7,{ }^{40}$ can override PMLIV-induced senescence in human fibroblasts. Here, we present evidence that MageA2 impairs p53 acetylation at NBs and blocks the ability of PMLIV to induce senescence in a normal cellular context. The exact contribution of MageA2 double targeting on p53 and on PMLIV for senescence regulation has not yet been possible to elucidate, because MageA2 deletion mutants losing the ability to bind p53 also lose PMLIV-binding capability probably due to conformational changes. In addition, functional PML and p53 are necessary for senescence induction, thus being impossible to analyze their contribution separately. The biological relevance of MageA2 was highlighted in a more physiological scenario such as the senescence induced by oncogenic signals. In such a context, expression of MageA2 could dampen the RasV12-induced senescence program by targeting primarily the PMLIV/p53 axis and by compromising the assembly of NBs.

PML-NBs have been suggested as important players for the formation of heterochromatin foci (SAHF) in senescence, ${ }^{20}$ hence it is possible to speculate that MageA2 by disturbing PML-NBs formation could affect in turn the formation of SAHFs.

Potentially oncogenic proliferative signals are able to interfere with a variety of growth-inhibitory processes, such as the induction of apoptosis, differentiation or senescence, each of which restrains neoplastic evolution. ${ }^{16}$ MAGE-A genes expression is an early event in tumorigenesis, mainly attributable to DNA hypomethylation, a process that accompanies and characterizes cell transformation. ${ }^{2,30}$ Deregulated activity of oncogenes such as ras appears to occur during the initial steps toward transformation; in such environment early expression of MageA2 could act in a cooperative functional interaction with oncogenic ras to overcome the PMLIV/p53 axis responsible for triggering cellular senescence, which constitutes one of the critical barriers for cellular transformation. Alternatively, in established tumors MageA2 could confer to cells the ability to survive and resist chemotherapy. ${ }^{10}$

Evidence presented here suggests that MageA2 expression can regulate critical steps in carcinogenesis and tumor progression. We highlight the potential contribution of MageA2 to override oncogene-induced senescence as an early tumor initiation process that, in addition to its anti-apoptotic effects, reflects the overall relevance of MageA2 in cancer.

\section{Materials and Methods}

Cell culture and reagents. U2OS, 293T, H1299 and Wi38 cell lines were obtained from the American Type Culture Collection (Rockville, MD, USA) and were cultured as recommended. 13923 melanoma cells have been described. ${ }^{12} \mathrm{PML}^{-/-}$
$\mathrm{MEF}^{21}$ and BJ/ET/RasV12ER cells ${ }^{29}$ were provided by G Del Sal (LNCIB, Trieste, Italy) and have been previously described.

TSA and 4-hydroxytamoxifen have been purchased from Sigma (St. Louis, MO, USA).

Plasmids, siRNA and transfections. Cells were transfected using Calcium Phosphate method for 293T cells, Lipofectamine 2000 Reagent (Invitrogen, Carlsbad, CA, USA) for $\mathrm{H} 1299$ and $\mathrm{PML}^{-1-}$ MEF, or FuGENE 6 (Roche, Mannheim, Germany) for U2OS cells, according to the manufacturer's instructions. Unless stated otherwise, cells were analyzed $24 \mathrm{~h}$ after transfection. HA-MageA1, -A2, -A4 and -A6 were cloned in pCDNA3 (Invitrogen). GST-MageA2, and GST-MageA4 were obtained by subcloning in pGEX-4T1 (GE Healthcare Biosciences, Buckinghamshire, UK). PCDNA3-PMLIV, PIG3-LUC, GFP-SUMO1; ${ }^{21}$ and Flag-HDAC3 ${ }^{10}$ have been previously described. pCS2-SUMO1 was from $\mathrm{L}$ Collavin (LNCIB, Trieste, Italy). Isoform-specific Flag-PML coding vectors were kindly provided by L Banks (ICGEB, Trieste, Italy). p300 was from B Amati (IFOM, Milan, Italy). MAGE-I silencing was previously described. ${ }^{10}$ Control siRNA (siC) targeted the bacterial LacZ gene (sequence $5^{\prime}$-GUGACCAGCGAAUACCUGU-3') and was purchased from Eurofins MWG operon (Ebersberg, Germany). Cells were transfected with siRNAs using Lipofectamine RNAiMAX Reagent (Invitrogen) as recommended by the manufacturer and analyzed after $48 \mathrm{~h}$.

Retroviral infections were performed by standard protocols (for details, see Supplementary Material and Methods). Retroviral plasmids used were: pWZLHygro-PMLIV (kindly provided by G Ferbeyre). pLPC-EGF-MageA2 and pLPCEGF-MageA4 were obtained by subcloning MageA2 and MageA4 cDNAs into pLPC-EGFP (Puromycin resistance).

Antibodies. Western blot analysis was performed according to the standard procedures using the following primary antibodies: for p53: D01, anti-p53 monoclonal (Santa Cruz Biotechnology, Santa Cruz, CA, USA), anti-acetyl p53 (Lys382) (Cell Signaling, Danvers, MA, USA); for Mage detection, affinity purified anti-MageA6 raised against GST-MageA6, anti-MageA monoclonal antibody (6C1-Santa Cruz Biotechnology). Other primary antibodies: anti-PML polyclonal $(\mathrm{H}-238)$ and monoclonal (PG-M3) antibodies and anti-p21 polyclonal antibody were from Santa Cruz Biotechnology; anti-SUMO1 monoclonal antibody (Zymed Laboratories, San Francisco, CA, USA), anti-acetylated lysine polyclonal antibody (Cell Signaling) and anti-p300 monoclonal antibody (BD Pharmingen, Franklin Lakes, NJ, USA); and antiactin polyclonal antibody (Sigma). For tags: anti-HA 12CA5 monoclonal antibody (Roche), anti-HA-7 and anti-Flag M2 monoclonal antibodies were from Sigma.

Immunofluorescence analysis. Cells were plated on glass coverslips in $3 \mathrm{~cm}$ culture dishes. After washing with PBS, cells were fixed in $3 \%$ paraformaldehyde (PFA) in PBS, treated with $1 \%$ glycine in PBS and permeabilized in $0.1 \%$ Triton $X-100$ in PBS. The staining was performed using specific antibodies incubated in $5 \%$ bovine serum albumin in PBS at $37^{\circ} \mathrm{C}$ followed by fluorescein isothiocyanate or tetramethylrhodamine isothiocyanate-conjugated secondary antibodies (Sigma) as indicated. Treatment with CSK buffer (100 mM $\mathrm{NaCl}, 300 \mathrm{mM}$ sucrose, $10 \mathrm{mM}$ Pipes, $3 \mathrm{mM} \mathrm{MgCl} 2,1 \mathrm{mM}$ EGTA, $15 \%$ glycerol, $1 \mathrm{mM}$ PMSF, plus $0.5 \%$ Triton $\mathrm{X}-100$ ) was performed for $10 \mathrm{~min}$ at $4{ }^{\circ} \mathrm{C}$ before fixation with PFA. SAHFs were revealed by Hoechst staining. Glass slides were analyzed using a laser scan confocal microscope (Zeiss, Thornwood, NY, USA) or an epifluorescence microscope (Leica, Mannheim, Germany). Images were obtained at $\times 100$ magnification.

Immunoprecipitation and in vitro pull-down. Cells were harvested in ice-cold lysis buffer containing $50 \mathrm{mM}$ Tris- $\mathrm{HCl} \mathrm{pH} 8.0,150 \mathrm{mM} \mathrm{NaCl}, 1 \% \mathrm{NP}-40$, $0.1 \mathrm{mM}$ sodium orthovanadate, $2 \mathrm{mM}$ DTT, $0.1 \mathrm{mM}$ PMSF, $5 \mathrm{mM}$ EDTA and Protease Inhibitor Cocktail (Sigma). After $10 \mathrm{~min}$ of rocking at $4{ }^{\circ} \mathrm{C}$, lysates were clarified by centrifugation and pre-cleared with $25 \mu$ lof Protein A-Sepharose CL-4B or GammaBind G-Sepharose (GE Healthcare Biosciences). Then, antibody prebound to $25 \mu \mathrm{l}$ of Protein A-Sepharose CL-4B or GammaBind G-Sepharose was added and incubated at $4{ }^{\circ} \mathrm{C}$ for $4 \mathrm{~h}$. The resin was then washed and bound proteins were eluted in SDS-PAGE sample buffer. For immunoprecipitation of endogenous $\mathrm{PML}$, cells were harvested in RIPA buffer containing $50 \mathrm{mM}$ Tris- $\mathrm{HCl} \mathrm{pH} 7.8$, $150 \mathrm{mM} \mathrm{NaCl}, 5 \mathrm{mM}$ EDTA, $0.5 \%$ Triton X-100, 0.5\% NP-40, 0.2\% deoxycholic acid, $0.1 \mathrm{mM}$ sodium orthovanadate, $0.1 \mathrm{mM}$ PMSF and protease inhibitor cocktail. For PML post-translational modifications analysis, RIPA buffer was supplemented with $10 \mathrm{mM} \mathrm{N}$-ethylmaleimide (Sigma) for sumoylation analysis, or $5 \mathrm{mM}$ butyric acid (Sigma) for acetylation analysis. Anti-PML monoclonal (PG-M3) antibody was prebound to Protein G-Sepharose and incubated with cells lysates for $16 \mathrm{~h}$ at $4^{\circ} \mathrm{C}$. For 
analysis of endogenous PML sumoylation, U2OS cells stably expressing FlagPMLIV were lysed in RIPA 200 buffer $(20 \mathrm{mM}$ Tris-HCl pH 7.4, $200 \mathrm{mM} \mathrm{NaCl}, 1 \mathrm{mM}$ EDTA, $0.5 \%$ NP40, $0.5 \%$ deoxycholic acid, $0.1 \%$ SDS, $0.1 \mathrm{mM}$ sodium orthovanadate, $0.1 \mathrm{mM}$ PMSF, Protease inhibitor cocktail and $10 \mathrm{mM} \mathrm{N}$-ethylmaleimide). Lysates were sonicated and then centrifugated $1 \mathrm{~min}$ at $2000 \mathrm{RPM}$. Anti-Flag monoclonal antibody was pre-bound to Protein G-Sepharose and incubated with cells lysates for $3 \mathrm{~h}$ at $4{ }^{\circ} \mathrm{C}$.

For in vitro binding assays ${ }^{35} \mathrm{~S}$-labeled proteins were in vitro translated using TNT Quick Coupled Transcription/Translation System (Promega, Madison, WI, USA) and incubated with purified GST, GST-MageA2 or GST-MageA4 (immobilized on glutathione-Sepharose 4B beads, GE Healthcare Biosciences) in pull-down buffer (PBS, 0.1\% NP-40, 10\% glycerol, $0.1 \mathrm{mM}$ PMSF and Protease Inhibitor Cocktail). Bound proteins were eluted and resolved by SDS-PAGE.

Transactivation assays. Cells were co-transfected in a 24-well plate with $100 \mathrm{ng}$ of PIG3LUC reporter constructs together with $35 \mathrm{ng}$ of pCDNA3-p53, either alone or with $150 \mathrm{ng}$ of pCDNA3-HA-MageAs or pCDNA3-PMLIV constructs. For normalization of transfection efficiency, $10 \mathrm{ng}$ of pRL-CMV reporter (Promega), constitutively expressing the Renilla reniformis luciferase, was included. After $24 \mathrm{~h}$, cells were lysed and assayed for luciferase activity using the Dual Luciferase kit (Promega).

BrdU incorporation. Cells were pulsed with $30 \mu \mathrm{M}$ bromodeoxyuridine (BrdU, Sigma) for $3 \mathrm{~h}$, then fixed with $3 \%$ PFA in PBS, permeabilized with $0.1 \%$ Triton X-100 in PBS, and RNA denatured with $50 \mathrm{mM} \mathrm{NaOH}$ for $20 \mathrm{~s}$. BrdU incorporation was measured by immunofluorescence using an anti-BrdU antibody (GE Healthcare Biosciences) and the nuclei were stained with Hoechst. Stained cells were visualized under a fluorescence microscope (Leica) and at least 200 cells were scored for BrdU incorporation in at least three independent experiments.

Analysis of SA- $\beta$-Gal. Cells were fixed in $0.5 \%$ glutaraldehyde in $\mathrm{PBS} / 2 \mathrm{mM}$ $\mathrm{MgCl}_{2}$ at $\mathrm{pH} 6.0$ and then stained with staining solution containing $5 \mathrm{mM} \mathrm{K}_{4} \mathrm{Fe}(\mathrm{CN})_{6}$ $5 \mathrm{mM} \mathrm{K}_{3} \mathrm{Fe}(\mathrm{CN})_{6}$ in $\mathrm{PBS} / 2 \mathrm{mM} \mathrm{MgCl}_{2}$ at pH 6.0 plus $1 \mathrm{mg} / \mathrm{ml} \mathrm{X-Gal,} \mathrm{for} 16 \mathrm{~h}$ at $37^{\circ} \mathrm{C}$. Cells were then washed extensively with $\mathrm{H}_{2} \mathrm{O}$ and observed under a bright field microscope.

Quantitative RT-PCR. Total RNA was extracted with Trizol reagent (Invitrogen) and cDNA was transcribed with a QuantiTect Reverse Transcription Kit (Qiagen, Hilden, Germany), according to the manufacturer's instructions. Realtime PCR was performed with SYBR Green PCR Master Mix (Applied Biosystems, Foster City, CA, USA) and StepOnePlus real time PCR machine (Applied Biosystems). Primer sequences are shown in the Supplementary Materials and Methods.

\section{Conflict of Interest}

The authors declare no conflict of interest.

Acknowledgements. We thank Andrea Bisso (LNCIB) for helpful discussion Marina Lusic (ICGEB) for advices and reagents, and Ramiro Mendoza Maldonado (ICGEB) for technical assistance with confocal acquisitions. This work was supported by grants from Associazione Italiana per la Ricerca sul Cancro (AIRC) IG4752 and AIRC Special Program Molecular Clinical Oncology "5 per mille", and PRIN 2006053543_003 to CS, and from ANPCyT (PICT07/00283) and CONICET (PIP/0674) to MM. LYP and MS are recipients of fellowships from AREA Science Park. MFL is recipient of fellowship from CONICET.

1. Chomez P, De Backer O, Bertrand M, De Plaen E, Boon T, Lucas S. An overview of the MAGE gene family with the identification of all human members of the family. Cancer Res 2001; 61: 5544-5551.

2. De Smet $C$, Lurquin $C$, Lethe $B$, Martelange $V$, Boon $T$. DNA methylation is the primary silencing mechanism for a set of germ line- and tumor-specific genes with a CpG-rich promoter. Mol Cell Biol 1999; 19: 7327-7335.

3. Kruit WH, van Ojik HH, Brichard VG, Escudier B, Dorval T, Dreno B et al. Phase 1/2 study of subcutaneous and intradermal immunization with a recombinant MAGE-3 protein in patients with detectable metastatic melanoma. Int J Cancer 2005; 117: 596-604

4. Caballero OL, Chen YT. Cancer/testis (CT) antigens: potential targets for immunotherapy. Cancer Sci 2009; 100: 2014-2021.
5. Sang $M$, Wang L, Ding C, Zhou X, Wang B, Lian $Y$ et al. Melanoma-associated antigen genes - an update. Cancer Lett 2011; 302: 85-90.

6. Laduron S, Deplus R, Zhou S, Kholmanskikh O, Godelaine D, De Smet C et al. MAGE-A1 interacts with adaptor SKIP and the deacetylase HDAC1 to repress transcription. Nucleic Acids Res 2004; 32: 4340-4350.

7. Zhu X, Asa SL, Ezzat S. Fibroblast growth factor 2 and estrogen control the balance of histone 3 modifications targeting MAGE-A3 in pituitary neoplasia. Clin Cancer Res 2008; 14: 1984-1996.

8. Kondo T, Zhu X, Asa SL, Ezzat S. The cancer/testis antigen melanoma-associated antigen-A3/A6 is a novel target of fibroblast growth factor receptor 2-IIlb through histone H3 modifications in thyroid cancer. Clin Cancer Res 2007; 13: 4713-4720.

9. Askew EB, Bai S, Hnat AT, Minges JT, Wilson EM. Melanoma antigen gene protein-A11 (MAGE-11) F-box links the androgen receptor NH2-terminal transactivation domain to p160 coactivators. J Biol Chem 2009; 284: 34793-34808.

10. Monte M, Simonatto M, Peche LY, Bublik DR, Gobessi S, Pierotti MA et al. MAGE-A tumor antigens target p53 transactivation function through histone deacetylase recruitment and confer resistance to chemotherapeutic agents. Proc Natl Acad Sci USA 2006; 103: 11160-11165.

11. Yang B, O'Herrin SM, Wu J, Reagan-Shaw S, Ma Y, Bhat KM et al. MAGE-A, mMage-b, and MAGE-C proteins form complexes with KAP1 and suppress p53-dependent apoptosis in MAGE-positive cell lines. Cancer Res 2007; 67: 9954-9962.

12. Marcar L, Maclaine NJ, Hupp TR, Meek DW. Mage-A cancer/testis antigens inhibit p53 function by blocking its interaction with chromatin. Cancer Res 2010; 70: 10362-10370.

13. Nagao T, Higashitsuji H, Nonoguchi K, Sakurai T, Dawson S, Mayer RJ et al. MAGE-A4 interacts with the liver oncoprotein gankyrin and suppresses its tumorigenic activity. J Biol Chem 2003; 278: 10668-10674.

14. Sakurai T, Itoh K, Higashitsuji $\mathrm{H}$, Nagao $\mathrm{T}$, Nonoguchi $\mathrm{K}$, Chiba $\mathrm{T}$ et al. A cleaved form of MAGE-A4 binds to Miz-1 and induces apoptosis in human cells. J Biol Chem 2004; 279: 15505-15514.

15. Peikert T, Specks U, Farver C, Erzurum SC, Comhair SA. Melanoma antigen A4 is expressed in non-small cell lung cancers and promotes apoptosis. Cancer Res 2006; 66: 4693-4700.

16. Hanahan D, Weinberg RA. Hallmarks of cancer: the next generation. Cell 2011; 144: 646-674.

17. Ferbeyre G, de Stanchina E, Querido E, Baptiste N, Prives C, Lowe SW. PML is induced by oncogenic ras and promotes premature senescence. Genes Dev 2000; 14: 2015-2027.

18. Pearson M, Carbone R, Sebastiani C, Cioce M, Fagioli M, Saito S et al. PML regulates p53 acetylation and premature senescence induced by oncogenic Ras. Nature 2000; 406: 207-210.

19. Bernardi R, Pandolfi PP. Structure, dynamics and functions of promyelocytic leukaemia nuclear bodies. Nat Rev Mol Cell Biol 2007; 8: 1006-1016.

20. Lallemand-Breitenbach V, de The H. PML nuclear bodies. Cold Spring Harb Perspect Biol 2010; 2: a000661.

21. Fogal V, Gostissa M, Sandy P, Zacchi P, Sternsdorf T, Jensen $\mathrm{K}$ et al. Regulation of p53 activity in nuclear bodies by a specific PML isoform. EMBO J 2000; 19: 6185-6195.

22. Bischof O, Kirsh O, Pearson M, Itahana K, Pelicci PG, Dejean A. Deconstructing PMLinduced premature senescence. EMBO J 2002; 21: 3358-3369.

23. Hayakawa F, Abe A, Kitabayashi I, Pandolfi PP, Naoe T. Acetylation of PML is involved in histone deacetylase inhibitor-mediated apoptosis. J Biol Chem 2008; 283: 24420-24425.

24. Guo A, Salomoni P, Luo J, Shih A, Zhong S, Gu W et al. The function of PML in p53dependent apoptosis. Nature Cell Biol 2000; 2: 730-736.

25. Vousden KH, Prives C. Blinded by the light: the growing complexity of p53. Cell 2009; 137 : 413-431.

26. Reineke EL, Kao HY. Targeting promyelocytic leukemia protein: a means to regulating PML nuclear bodies. Int J Biol Sci 2009; 5: 366-376.

27. Nichol JN, Petruccelli LA, Miller Jr WH. Expanding PML's functional repertoire through post-translational mechanisms. Front Biosci 2009; 14: 2293-2306.

28. Zhong S, Muller S, Ronchetti S, Freemont PS, Dejean A, Pandolfi PP. Role of SUMO-1modified PML in nuclear body formation. Blood 2000; 95: 2748-2752.

29. Drost J, Mantovani F, Tocco F, Elkon R, Comel A, Holstege H et al. BRD7 is a candidate tumour suppressor gene required for p53 function. Nature Cell Biol 2010; 12: 380-389.

30. Simpson AJ, Caballero OL, Jungbluth A, Chen YT, Old LJ. Cancer/testis antigens, gametogenesis and cancer. Nat Rev Cancer 2005; 5: 615-625.

31. Liu W, Cheng S, Asa SL, Ezzat S. The melanoma-associated antigen A3 mediates fibronectincontrolled cancer progression and metastasis. Cancer Res 2008; 68: 8104-8112.

32. Atanackovic D, Hildebrandt $Y$, Jadczak A, Cao Y, Luetkens T, Meyer S et al. Cancer-testis antigens MAGE-C1/CT7 and MAGE-A3 promote the survival of multiple myeloma cells. Haematologica 2010; 95: 785-793.

33. Aprelikova O, Pandolfi S, Tackett S, Ferreira M, Salnikow K, Ward Y et al. Melanoma antigen-11 inhibits the hypoxia-inducible factor prolyl hydroxylase 2 and activates hypoxic response. Cancer Res 2009; 69: 616-624.

34. Suzuki T, Yoshida K, Wada Y, Hamai Y, Sentani K, Oue N et al. Melanoma-associated antigen-A1 expression predicts resistance to docetaxel and paclitaxel in advanced and recurrent gastric cancer. Oncol Rep 2007; 18: 329-336.

35. Wu WS, Vallian S, Seto E, Yang WM, Edmondson D, Roth S et al. The growth suppressor PML represses transcription by functionally and physically interacting with histone deacetylases. Mol Cell Biol 2001; 21: 2259-2268.

36. Lallemand-Breitenbach V, Zhu J, Puvion F, Koken M, Honore N, Doubeikovsky A et al. Role of promyelocytic leukemia (PML) sumolation in nuclear body formation, 11S 
proteasome recruitment, and As203-induced PML or PML/retinoic acid receptor alpha degradation. J Exp Med 2001; 193: 1361-1371.

37. Lallemand-Breitenbach V, Jeanne M, Benhenda S, Nasr R, Lei M, Peres L et al. Arsenic degrades PML or PML-RARalpha through a SUMO-triggered RNF4/ubiquitin-mediated pathway. Nature Cell Biol 2008; 10: 547-555.

38. Doyle JM, Gao J, Wang J, Yang M, Potts PR. MAGE-RING protein complexes comprise a family of E3 ubiquitin ligases. Mol Cell 2010; 39: 963-974.
39. Langley E, Pearson M, Faretta M, Bauer UM, Frye RA, Minucci S et al. Human SIR2 deacetylates p53 and antagonizes PML/p53-induced cellular senescence. EMBO J 2002; 21: 2383-2396.

40. Bischof O, Nacerddine K, Dejean A. Human papillomavirus oncoprotein E7 targets the promyelocytic leukemia protein and circumvents cellular senescence via the $\mathrm{Rb}$ and $\mathrm{p} 53$ tumor suppressor pathways. Mol Cell Biol 2005; 25 : 1013-1024.

Supplementary Information accompanies the paper on Cell Death and Differentiation website (http://www.nature.com/cdd) 